\title{
Methanol Electro-oxidation on Pt Surface: Revisiting the Cyclic Voltammetry Interpretation
}

Dong Young Chung, ${ }^{\#, \dagger,+}$ Kyung-Jae Lee $e^{\#, \dagger,+, \uparrow}$ and Yung-Eun Sung ${ }^{*, \dagger, *}$

${ }^{\dagger}$ Center for Nanoparticle Research, Institute for Basic Science (IBS), Seoul 151-747, South Korea

${ }^{\ddagger}$ School of Chemical and Biological Engineering, Seoul National University, Seoul 151-747, South Korea

\# These authors contributed equally to this work.

\section{Corresponding Author}

E-mail: ysung@snu.ac.kr 
We proposed conceptual equivalent circuit, as in Fig. 8, for the methanol oxidation reaction. The EIS fitting was conducted using the equivalent circuit, and the complex methanol oxidation reaction in region 2 to 4 were analyzed quantitatively. To avoid interpretation with our own intensions, however, we used modified equivalent circuit based on some assumptions below, and conducted EIS fitting; Two RC parallel circuits in parallel could be summarized into one RC parallel circuit. In modified equivalent circuit, therefore, two RC parallel circuits in parallel are replaced with one RC parallel circuit. In addition, one RC parallel circuit is added in series to the existing equivalent circuit to analyze small semicircle in high frequency region, which could be originated from porous electrode structure or the counter electrode (Fig. S1). The fitting results are shown in Fig. S3, and the values are recorded in Table S1..

The potential in region 2 is higher than the theoretical potential for the methanol oxidation, which means that the methanol oxidation reaction could occur easily in region 2 . Therefore, we could expect that the methanol oxidation reaction at free $\mathrm{Pt}$ surface (reaction 1 in manuscript) would take place more easily along the potential increases, and confirmed that $\mathrm{R} 1$ which is related to reaction 1 decreased as the potential increased, as expected. In addition, $\mathrm{R} 3$, which accounts for the reaction with intermediate, $\mathrm{CO}$ and $\mathrm{OH}$ (reaction 3 in manuscript) also decreased as the potential increased. This decreasing R3 along the potential increases, i.e. enhancing the kinetics of reaction 3, is consistent with the fact that the peak at low frequency region shifts toward high frequency. (Region 2, $0.60 \sim 0.70 \mathrm{~V}$ ) At $0.75 \mathrm{~V}$, however, R1 and R3 both increased abruptly, as Ref. 27 in manuscript already reported. As the increased amount of the steady-state current in Fig 4 between $0.70 \sim 0.80 \mathrm{~V}$ decreased when compared to that between $0.60 \sim 0.70 \mathrm{~V}$, we could infer that the environment of methanol oxidation reaction changed along this potential region.

$\mathrm{R} 1$ became a negative value in region 3 . As the resistance means the changes in faradaic current along the potential changes $\left(R=\frac{\partial E}{\partial i_{F}}\right)$, the negative resistance implies that the faradaic current would decrease as the potential increases. This fluctuating tendency of R1 could be interpreted with the coverage of free Pt surface and $\mathrm{OH}$; According to ref 27, $\mathrm{OH}$ coverage on Pt surface increases along the potential increases. In early region $2(0.60 \sim 0.70$ $\mathrm{V}), \mathrm{OH}$ coverage increases, and it helps to remove $\mathrm{CO}_{\mathrm{ad}}$ on $\mathrm{Pt}$ surface, result in enhancing reaction 1 due to newly exposed free Pt surface. As a result, R1 decreases in early region 2 . As $\mathrm{OH}$ coverage on $\mathrm{Pt}$ surface further increases $(0.75 \mathrm{~V})$, however, the effect of decreasing 
free Pt surface became more dominant. Thus, reaction 1 became slow and R1 increases consequently. As the effect of decreasing free Pt coverage and increasing $\mathrm{OH}$ coverage on $\mathrm{Pt}$ surface intensified, the faradaic current decreased along the potential increases resulting to the negative resistance. It is supported by the following facts; the peak in low frequency region of Bode plot shifts toward high frequency region in region 2, and there is an abrupt phase change in region 3 . In addition, the steady-state current decreases along the potential increases after $0.80 \mathrm{~V} .\left(R=\frac{\partial E}{\partial i_{F}}<0\right)$

The increase of $\mathrm{R} 3$ in late region 2 is attributed to the decrease of reaction 1 ; reaction 1 causes $\mathrm{Pt}-\mathrm{CO}_{\mathrm{ad}}$, and it reacts with $\mathrm{OH}$. As mentioned above, the Pt-CO coverage decreases along the potential increases, thus, reaction 3 also became slow and resulted in decrease of R3.

In region 4 , the reaction 1 is rate-determining step, like region 3. As reaction 1 became hard due to the problem with free Pt coverage along the increasing potential, therefore R1 keep increasing in $0.90 \sim 0.95 \mathrm{~V}$. The slowdown of reaction 1 also gives rise to the retardation of reaction 3, thus, R3 also keep increases. Suddenly, however, the absolute value of R1 and R3 decrease above $0.95 \mathrm{~V}$, and these behaviors are not clearly understood yet. Nevertheless, the phenomena, such as the shift of the Nyquist plot from $2^{\text {nd }}$ quadrant to $4^{\text {th }}$ quadrant, and occurrence of new peak in Bode plot suggest that there is new faradaic reaction happened.

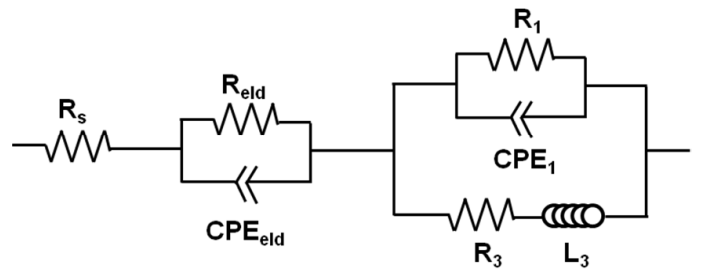

Fig. S1 The modified equivalent circuit for quantitative analysis 


\begin{tabular}{c|c|c|c|c|c|c|c|c|c|c}
\hline Region & Potential (V) & Rs & R_eld & CPE_eld-T & CPE_eld-P & R1 & CPE1-T & CPE1-T & R3 & L3 \\
\hline \multirow{4}{*}{ II } & 0.60 & 13.23 & 5.92 & $7.20 \mathrm{E}-05$ & 0.595 & 115.4 & 0.003 & 0.879 & 50.5 & 1886.0 \\
\cline { 2 - 11 } & 0.65 & 13.82 & 5.10 & $3.84 \mathrm{E}-05$ & 0.669 & 79.16 & 0.004 & 0.809 & 42.5 & 442.2 \\
\cline { 2 - 12 } & 0.70 & 14.89 & 4.3 & $1.11 \mathrm{E}-05$ & 0.760 & 54.18 & 0.005 & 0.729 & 16.5 & 144.9 \\
\cline { 2 - 12 } & 0.75 & 13.59 & 5.59 & $7.13 \mathrm{E}-05$ & 0.608 & 618.2 & 0.004 & 0.810 & 24.7 & 201.1 \\
\hline \multirow{4}{*}{ III } & 0.80 & 13.8 & 5.77 & 0.00015 & 0.556 & -160.4 & 0.003 & 0.882 & 42.0 & 241.4 \\
\cline { 2 - 11 } & 0.85 & 13.8 & 5.42 & $5.03 \mathrm{E}-05$ & 0.641 & -109.4 & 0.003 & 0.838 & 97.7 & 156.8 \\
\hline \multirow{5}{*}{ IV } & 0.90 & 14.63 & 4.54 & $1.58 \mathrm{E}-05$ & 0.760 & -198.4 & 0.003 & 0.816 & 309.9 & 310.6 \\
\cline { 2 - 11 } & 0.93 & 15.05 & 4.06 & $5.58 \mathrm{E}-06$ & 0.851 & -263.9 & 0.003 & 0.780 & 389.5 & 177.9 \\
\cline { 2 - 11 } & 0.95 & 14.17 & 5.37 & $3.29 \mathrm{E}-05$ & 0.670 & -774.4 & 0.003 & 0.854 & 1054.0 & 509.6 \\
\cline { 2 - 11 } & 0.965 & 14.61 & 4.97 & $2.32 \mathrm{E}-05$ & 0.713 & -502.8 & 0.003 & 0.843 & 557.7 & 148.8 \\
\cline { 2 - 11 } & 0.975 & 14.68 & 4.44 & $1.17 \mathrm{E}-05$ & 0.783 & -258.9 & 0.003 & 0.809 & 262.7 & 47.0 \\
\hline
\end{tabular}

Table. S1 Fitting results 
(a)

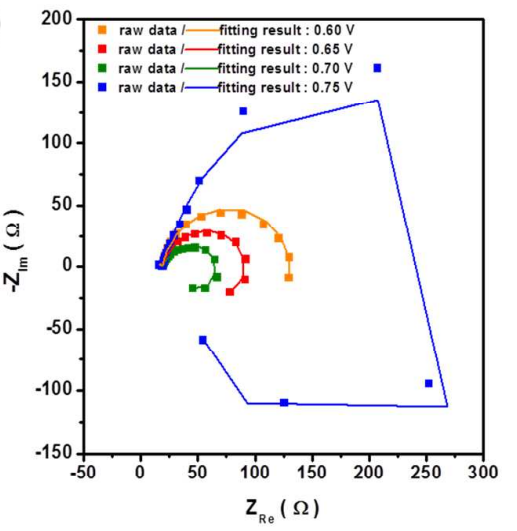

(b)

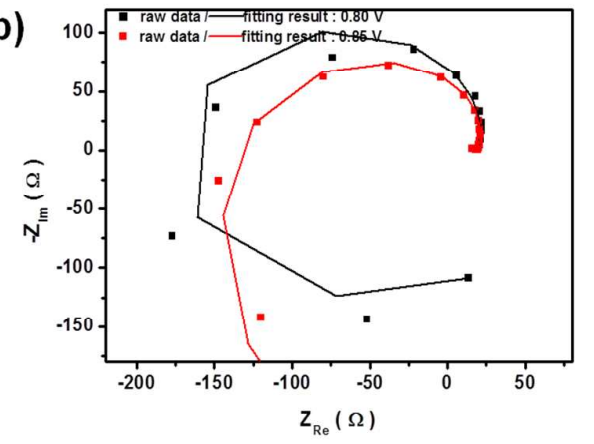

(c)

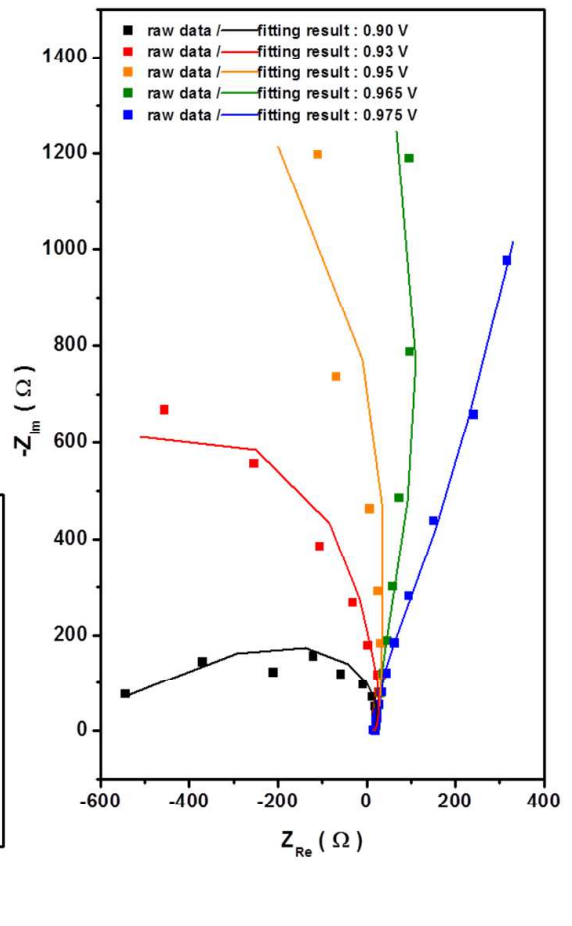

Fig. S2 Raw data and fitting results 